\title{
Multi-material topology optimization of laminated composite beam cross sections
}

\author{
Blasques, José Pedro Albergaria Amaral; Stolpe, Mathias
}

Published in:

Composite Structures

Link to article, DOI:

10.1016/j.compstruct.2012.05.002

Publication date:

2012

Link back to DTU Orbit

Citation (APA):

Blasques, J. P. A. A., \& Stolpe, M. (2012). Multi-material topology optimization of laminated composite beam cross sections. Composite Structures, 94, 3278-3289. https://doi.org/10.1016/j.compstruct.2012.05.002

\section{General rights}

Copyright and moral rights for the publications made accessible in the public portal are retained by the authors and/or other copyright owners and it is a condition of accessing publications that users recognise and abide by the legal requirements associated with these rights.

- Users may download and print one copy of any publication from the public portal for the purpose of private study or research.

- You may not further distribute the material or use it for any profit-making activity or commercial gain

- You may freely distribute the URL identifying the publication in the public portal

If you believe that this document breaches copyright please contact us providing details, and we will remove access to the work immediately and investigate your claim. 


\section{Accepted Manuscript}

Multi-material topology optimization of laminated composite beam cross sections

José Pedro Blasques, Mathias Stolpe

PII: S0263-8223(12)00208-5

DOI: http://dx.doi.org/10.1016/j.compstruct.2012.05.002

Reference: COST 4634

To appear in:

Composite Structures

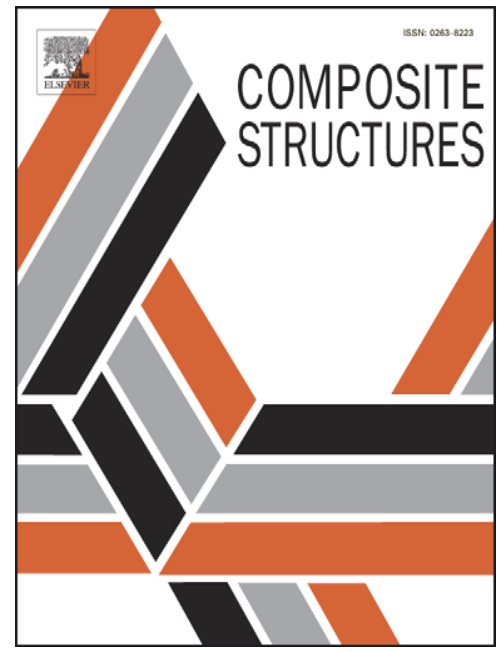

Please cite this article as: Blasques, J.P., Stolpe, M., Multi-material topology optimization of laminated composite beam cross sections, Composite Structures (2012), doi: http://dx.doi.org/10.1016/j.compstruct.2012.05.002

This is a PDF file of an unedited manuscript that has been accepted for publication. As a service to our customers we are providing this early version of the manuscript. The manuscript will undergo copyediting, typesetting, and review of the resulting proof before it is published in its final form. Please note that during the production process errors may be discovered which could affect the content, and all legal disclaimers that apply to the journal pertain. 


\title{
Multi-material topology optimization of laminated composite beam cross sections
}

\author{
José Pedro Blasques ${ }^{\mathrm{a}, 1}$, Mathias Stolpe ${ }^{\mathrm{a}}$ \\ ${ }^{a}$ DTU Wind Energy, Technical University of Denmark, Frederiksborgvej 399, P.O. Box 49, Building 114, 4000 Roskilde, Denmark
}

\begin{abstract}
This paper presents a novel framework for simultaneous optimization of topology and laminate properties in structural design of laminated composite beam cross sections. The structural response of the beam is evaluated using a beam finite element model comprising a cross section analysis tool which is suitable for the analysis of anisotropic and inhomogeneous sections of arbitrary geometry. The optimization framework is based on a multi-material topology optimization model in which the design variables represent the amount of the given materials in the cross section. Existing material interpolation, penalization, and filtering schemes have been extended to accommodate any number of anisotropic materials. The methodology is applied to the optimal design of several laminated composite beams with different cross sections. Solutions are presented for a minimum compliance (maximum stiffness) problem with constraints on the weight, and the shear and mass center positions. The practical applicability of the method is illustrated by performing optimal design of an idealized wind turbine blade subjected to static loading of aerodynamic nature. The numerical results suggest that the proposed framework is suitable for simultaneous optimization of cross section topology and identification of optimal laminate properties in structural design of laminated composite beams.
\end{abstract}

Keywords: Beams, Cross section analysis, Laminated composites, Multi-material topology optimization

\section{Introduction}

This paper describes a methodology for simultaneous optimization of the topology and the laminate properties of beam cross sections. The objective in the considered optimal design problems is to minimize the compliance of the beam, i.e., to maximize stiffness, with constraints on the weight and the shear and mass center positions. The design variables represent the volume fractions of each of a predefined set of candidate materials in the cross section. The structural response of the beam is computed using a beam finite element model which incorporates an efficient and accurate cross section analysis tool able to account for the effects of material anisotropy and inhomogeneity in sections of arbitrary geometry.

The work presented here is based on the developments in the field of multi-material topology optimization, see e.g., Bendsøe and Sigmund [1] and Sigmund and Torquato [2]. The work is also inspired by the ideas for optimal design of composite structures suggested by Lund and Stegmann [3] and Stegmann and Lund [4]. We follow a common approach in topology optimization and replace the discrete design variables by continuous variables and attempt to force the variables to their bounds by an appropriate material model. The model is based on an extension of the SIMP material interpolation scheme to problems with multiple (anisotropic) materials. The reader is referred to Bendsøe and Sigmund [1? ] for an overview of material interpolation schemes.

\footnotetext{
Email addresses: jpbl@risoe.dtu.dk (José Pedro Blasques), matst@dtu.dk (Mathias Stolpe)

${ }^{1}$ Corresponding author. Phone: +45 21798854
}

Certain problems in topology optimization suffer from undesirable features such as mesh-dependency and checkerboard patterns in the design, see e.g. Sigmund and Petersson [6]. These issues can be resolved by regularization of the problem, for example by filtering of the design variables as described in Bourdin [7]. The filtering technique of Bruns and Tortorelli [8] is here extended to multi-material problems and utilized to avoid mesh-dependency and the appearance of checkerboard patterns. Analytical expressions for the gradients of the cross section stiffness parameters and shear and mass center position are derived. The optimal design problems can therefore be solved using any modern and robust numerical optimization method for continuous constrained optimization.

The proposed methodology is applied to the design of laminated composite beams. Several numerical results are presented which illustrate the ability of the method in solving the problem of identifying the optimal cross section topology and the material properties. The applicability of the method is attested by the results presented for the maximum stiffness optimization of an idealized wind turbine rotor blade cross section with prescribed weight and, shear and mass center placement constraints.

Several approaches have been presented in the literature for optimal design of beams combining different structural models and optimization problems. A methodology combining a beam finite element model and a cross section analysis tool was presented by Blasques and Stolpe [9] for maximum stiffness and minimum weight design of laminated composite beams. The three dimensional variation of laminate thickness, stacking sequence, and fiber orientation of several composite beams with different cross section shapes were optimized. Based on the 
same cross section analysis tool, Li et al. [10] optimized the cross sectional stiffness and inertia properties of a helicopter rotor blade. Similarly, the work by Ganguli and Chopra [11] and Murugan and Ganguli [12] addressed optimal design of laminated composite beams with stiffness and aeroelastic constraints. The design variables were the fiber orientations of the laminate on the walls of the internal blade spar.

The references in the preceding paragraph all assumed that the topology of the beam was to a large extent fixed and the problem consisted mostly of optimizing the laminate properties. That is, the optimal design of the cross section topology or structural lay-out was not fully addressed. The work by $\mathrm{Kim}$ and $\mathrm{Kim}[13,14]$ is perhaps the first using topology optimization in optimal design of the structural lay-out of beam cross sections. Their work addresses the design of beam cross sections to simultaneously increase torsional stiffness and reduce cross section distortion. Donoso and Sigmund [15] have used a similar approach to optimize the cross section topology and maximize the cross section distortion to produce "warping mechanisms".

The references in the previous paragraph consider only isotropic materials and do not address optimization of material or laminate properties. The methodology described in this paper allows for the simultaneous optimization of the cross section topology and distribution of given (anisotropic) materials or laminate properties.

Beam finite elements are built on specific assumptions and suitable only for the analysis of relatively long and slender structures. These assumptions allow for a significant reduction in the size of the resulting global finite element matrices. This type of analysis model is therefore attractive in applications requiring a large number of evaluations of the equilibrium equations such as wind turbine aeroelastic analysis codes (see, e.g., Hansen et al. [16]). When combined with advanced cross section analysis techniques, it is possible to obtain accurate estimates of both the global response of the beam and, to a certain extent, the three dimensional stress field.

Reviews on the different cross section analysis tools described in the literature are presented by Jung et al. [17] and Volovoi et al. [18]. Probably the most comprehensive work in this field is that by Hodges [19] and co-workers ( $\mathrm{Yu}$ and Hodges [20]). The beam formulation builds on the Variational Asymptotic Method (VAM) which was first used in this context by Berdichevsky [21]. The theory has served as the basis for the development of the Variational Asymptotic Beam Section analysis tool (VABS) described and validated in $\mathrm{Yu}$ and Hodges [20] and Volovoi et al. [22].

The cross section analysis tool used in this paper is based on the theory introduced by Giavotto et al. [23], and further detailed by Ghiringelli and Mantegazza [24], and Ghiringelli [25]. The formulation is based on the Saint-Venant's principle where only solutions away from the ends of the beam are considered. The cross section warping displacements are approximated using a finite element discretization of the cross section geometry. The formulation is able to account for the effects of material anisotropy and inhomogeneity in cross sections of arbitrary geometry. The theory has been implemented in the open source software BECAS - BEam Cross section Analysis Software (Blasques [26]). BECAS has been validated against VABS and exactly the same magnitude of the cross section stiffness parameters have been obtained for a series of cases including, anisotropic, inhomogeneous, closed, open, and multicelled, cross sections (Blasques [26]). Effects like tapering, pretwist, and curvature are not yet accounted for.

\section{Structural model}

A formulation for a linear elastic beam finite element of arbitrary section geometry accounting for the effects of material anisotropy and inhomogeneity is presented here. The analysis of the cross section properties is described first. Finally, the beam finite element equations for static equilibrium are presented next. The devised model is based on the assumptions that the loads are static, displacements are small, and that the material is linear elastic.

\subsection{Cross section analysis}

The theory presented in this section assumes that the beam structure is relatively long and slender and does not present abrupt variations in cross section geometry, load distribution and material properties along its length. Consequently, the resulting displacement and strain gradients along the beam axis are assumed to be relatively small. The assumptions mentioned before are not imposed along the cross section coordinates in the cross section plane. For clarity, the notation throughout this section has been kept close to that adopted by Giavotto et al. in [23]. The implementation of the theory into the cross section analysis tool BECAS is detailed in Blasques [26].

\subsubsection{General properties}

Let us consider a slice $d z$ of a beam. The strain and stresses acting at a point in the slice representing the cross

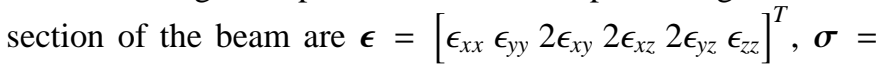
$\left[\sigma_{x x} \sigma_{y y} \sigma_{x y} \sigma_{x z} \sigma_{y z} \sigma_{z z}\right]^{T}$ which are related by the stress-strain relation or Hooke's law as $\sigma=\mathbf{Q} \epsilon$. In this case, $\mathbf{Q}$ is the material constitutive matrix.

The internal forces $\boldsymbol{\theta}=\left[\mathbf{T}^{T} \mathbf{M}^{T}\right]^{T}$ acting at a beam section are described in Figure 1(a). The components of the force vector $\mathbf{T}=\left[T_{x} T_{y} T_{z}\right]^{T}$ consist of the transverse forces $T_{x}$ and $T_{y}$ acting in the plane of the section, and the tension force $T_{z}$. The moment vector $\mathbf{M}=\left[M_{x} M_{y} M_{z}\right]^{T}$ is defined by the bending moment components $M_{x}$ and $M_{y}$, and the torsion moment $M_{z}$. These forces and moments are statically equivalent to the stress components $\mathbf{p}=\left[\sigma_{x z} \sigma_{y z} \sigma_{z z}\right]^{T}$ acting on the section face and defined as $\boldsymbol{\theta}=\int_{A} \mathbf{Z}^{T} \mathbf{p}$ dA where the matrix $\mathbf{Z}=\left[\mathbf{I}_{3} \mathbf{n}^{T}\right], \mathbf{I}_{3}$ is an identity matrix of size $3 \times 3, A$ is the cross section area, and

$$
\mathbf{n}=\left[\begin{array}{ccc}
0 & -z & y \\
z & 0 & -x \\
-y & x & 0
\end{array}\right]
$$

The coordinates $x$ and $y$ in the section plane are given with respect to the cross section reference point $O$ (cf. Figure 1). 
The cross section deformations associated with the load vector $\boldsymbol{\theta}$ are described by the cross section strain-curvature vector $\boldsymbol{\psi}=\left[\boldsymbol{\tau}^{T} \boldsymbol{\kappa}^{T}\right]^{T}$ (as described in Figure 1(b)). The shear component $\tau=\left[\tau_{x} \tau_{y} \tau_{z}\right]^{T}$ is composed of the shear strains $\tau_{x}$ and $\tau_{y}$, and the tension strain $\tau_{z}$. The components of the vector of curvatures $\boldsymbol{\kappa}=\left[\begin{array}{lll}\kappa_{x} & \kappa_{y} & \kappa_{z}\end{array}\right]^{T}$ are the bending curvatures $\kappa_{x}$ and $\kappa_{y}$, and the twist rate $\kappa_{z}$. The section forces and moments in $\boldsymbol{\theta}$, and the strains and curvatures in $\psi$ are related through the constitutive relation $\boldsymbol{\psi}=\mathbf{F}_{s} \boldsymbol{\theta}$ where $\mathbf{F}_{s}$ is the $6 \times 6$ cross section compliance matrix. For most practical applications $\mathbf{F}_{s}$ is symmetric positive definite. Hence $\mathbf{K}_{s}=\mathbf{F}_{s}^{-1}$, where $\mathbf{K}_{s}$ is the cross section stiffness matrix, and consequently the following relation holds $\boldsymbol{\theta}=\mathbf{K}_{s} \boldsymbol{\psi}$.

A methodology is presented in the next sections for the estimation of the parameters in $\mathbf{F}_{s}$. This formulation accounts for effects stemming from material anisotropy and inhomogeneity and is valid for cross sections of arbitrary geometry.

\subsubsection{Beam kinematics and finite element formulation}

The total displacement $\mathbf{s}=\left[\begin{array}{lll}s_{x} & s_{y} & s_{z}\end{array}\right]^{T}$ of a point in the cross section is

$$
\mathbf{s}=\mathbf{v}+\mathbf{g}
$$

where the displacement components $\mathbf{v}=\left[\begin{array}{lll}v_{x} & v_{y} & v_{z}\end{array}\right]^{T}$ are associated with the rigid body translations and rotations of the cross section. Assuming small displacements and rotations,

$$
\mathbf{v}=\mathbf{Z r}
$$

where $\mathbf{r}=\left[\begin{array}{ll}\chi^{T} & \varphi^{T}\end{array}\right]^{T}$. The components $\chi=\left[\begin{array}{lll}\chi_{x} & \chi_{y} & \chi_{z}\end{array}\right]^{T}$ represent the translations of the cross section reference point, while $\boldsymbol{\varphi}=\left[\varphi_{x} \varphi_{y} \varphi_{z}\right]^{T}$ are the cross section rotations.

Finally, $\mathbf{g}=\left[\begin{array}{lll}g_{x} & g_{y} & g_{z}\end{array}\right]^{T}$ is the displacement vector associated with the in- and out-of-plane cross section distortion or warping. Assume that the cross section geometry is discretized using, e.g., two dimensional isoparametric finite elements. In this case, the three dimensional warping displacements $\mathbf{g}$ can be approximated as

$$
\mathbf{g}(x, y) \approx \mathbf{N}(x, y) \mathbf{u}
$$

where $\mathbf{N}$ is the matrix of finite element shape functions and $\mathbf{u}$ the nodal warping displacements. Inserting (3) and (4) in in (2) yields

$$
\mathbf{s}=\mathbf{Z r}+\mathbf{N u}
$$

Assuming small displacements, the strain-displacement relation is $\epsilon_{\alpha \beta}=1 / 2\left(\partial s_{\alpha} / \partial \beta+\partial s_{\beta} / \partial \alpha\right),(\alpha, \beta=x, y, z)$. Hence, the three dimensional strain field $\boldsymbol{\epsilon}$ at the cross section level is

$$
\boldsymbol{\epsilon}=\mathbf{S Z} \boldsymbol{\psi}+\mathbf{B N u}+\mathbf{S N} \frac{\partial \mathbf{u}}{\partial z}
$$

The strain-displacement matrices $\mathbf{B}$ and $\mathbf{S}$ are defined as

$$
\mathbf{B}=\left[\begin{array}{cccccc}
\partial / \partial x & 0 & \partial / \partial y & 0 & 0 & 0 \\
0 & \partial / \partial y & \partial / \partial x & 0 & 0 & 0 \\
0 & 0 & 0 & \partial / \partial x & \partial / \partial y & 0
\end{array}\right]^{T}
$$

and $\mathbf{S}=\left[\begin{array}{ll}\mathbf{0} & \mathbf{I}_{3}\end{array}\right]$, where $\mathbf{0}_{3}$ and $\mathbf{I}_{3}$ are the $3 \times 3$ zero and identity matrices, respectively. The derivatives $\partial / \partial z$ along the length of the beam have been conveniently separated and left unsolved. The vector of strains and curvatures $\psi=\left[\tau^{T} \kappa^{T}\right]^{T}$ (cf. Figure $1(b))$ is defined in function of the rigid body motions, $\mathbf{r}$, as

$\boldsymbol{\psi}=\left(\mathbf{T}_{r}+\frac{\partial}{\partial z}\right) \mathbf{r}$, where $\mathbf{T}_{r}=\left[\begin{array}{ll}\mathbf{0}_{3} & \mathbf{t}_{r} \\ \mathbf{0}_{3} & \mathbf{0}_{3}\end{array}\right], \mathbf{t}_{r}=\left[\begin{array}{ccc}0 & -1 & 0 \\ 1 & 0 & 0 \\ 0 & 0 & 0\end{array}\right]$,

The resulting strain and curvature components in (6) are recognizable from typical beam theory and given as $\tau_{x}=\partial \chi_{x} / \partial z-\varphi_{y}$, $\tau_{y}=\partial \chi_{y} / \partial z+\varphi_{x}, \tau_{z}=\partial \chi_{x} / \partial z, \kappa_{x}=\partial \varphi_{x} / \partial z, \kappa_{y}=\partial \varphi_{y} / \partial z$, and $\kappa_{z}=\partial \varphi_{z} / \partial z$.

\subsubsection{Principle of virtual displacements}

According to the principle of virtual displacements, at equilibrium, the total external virtual work per unit length has to be equal to the total internal virtual work per unit length for any compatible, small virtual displacements. The total external virtual work per unit length is stemming from the work done by the section stresses $\mathbf{p}$ going through the virtual displacements $\delta$ s. The total internal virtual work per unit length or the elastic strain energy of the cross section is defined as the work done by the stresses $\sigma$ along the virtual strains $\delta \epsilon$ (associated with the virtual displacements). After manipulation (see Blasques [26]), the following set of equations describing the response of the cross section are obtained

$$
\left\{\begin{array}{r}
\mathbf{M} \frac{\partial^{2} \mathbf{u}}{\partial z^{2}}+\left(\mathbf{C}-\mathbf{C}^{T}\right) \frac{\partial \mathbf{u}}{\partial z}+\mathbf{L} \frac{\partial \psi}{\partial z}-\mathbf{E u}-\mathbf{R} \psi=\mathbf{0} \\
\mathbf{L}^{T} \frac{\partial \mathbf{u}}{\partial z}+\mathbf{R}^{T} \mathbf{u}+\mathbf{A} \boldsymbol{\psi}=\boldsymbol{\theta} \\
\frac{\partial \boldsymbol{\theta}}{\partial z}=\mathbf{T}_{r}^{T} \boldsymbol{\theta}
\end{array}\right.
$$

Each of the system matrices presented above is defined as

$$
\begin{aligned}
\underset{(6 \times 6)}{\mathbf{A}} & =\sum_{e=1}^{n_{e}} \int_{A} \mathbf{Z}_{e}^{T} \mathbf{S}_{e}^{T} \mathbf{Q}_{e} \mathbf{S}_{e} \mathbf{Z}_{e} \mathrm{dA}, \underset{\left(n_{d} \times 6\right)}{\mathbf{R}}=\sum_{e=1}^{n_{e}} \int_{A} \mathbf{N}_{e}^{T} \mathbf{B}_{e}^{T} \mathbf{Q}_{e} \mathbf{S}_{e} \mathbf{Z}_{e} \mathrm{dA} \\
\underset{\left(n_{d} \times n_{d}\right)}{\mathbf{E}} & =\sum_{e=1}^{n_{e}} \int_{A} \mathbf{N}_{e}^{T} \mathbf{B}_{e}^{T} \mathbf{Q}_{e} \mathbf{B}_{e} \mathbf{N}_{e} \mathrm{dA}, \underset{\left(n_{d} \times n_{d}\right)}{\mathbf{C}}=\sum_{e=1}^{n_{e}} \int_{A} \mathbf{N}_{e}^{T} \mathbf{B}_{e}^{T} \mathbf{Q}_{e} \mathbf{S}_{e} \mathbf{N}_{e} \mathrm{dA} \quad(8) \\
\underset{\left(6 \times n_{d}\right)}{\mathbf{L}} & =\sum_{e=1}^{n_{e}} \int_{A} \mathbf{Z}_{e}^{T} \mathbf{S}_{e}^{T} \mathbf{Q}_{e} \mathbf{S}_{e} \mathbf{N}_{e} \mathrm{dA}, \underset{\left(n_{d} \times n_{d}\right)}{\mathbf{M}}=\sum_{e=1}^{n_{e}} \int_{A} \mathbf{N}_{e}^{T} \mathbf{S}_{e}^{T} \mathbf{Q}_{e} \mathbf{S}_{e} \mathbf{N}_{e} \mathrm{dA}
\end{aligned}
$$

where $e$ is the element number and $n_{e}$ is the number of finite elements in the cross section mesh. The total number of degrees of freedom associated with the cross section finite element mesh is $n_{d}=n_{n} \times 3$ where the number of nodes $n_{n}$ multiplies the number of degrees of freedom at each node - the three dimensional nodal displacements $u_{x}, u_{y}$, and $u_{z}$. The sums in (8) refer to the typical assembly procedure used in finite element analysis. The finite element matrices can be successfully built using, e.g., four node isoparametric finite elements.

The second order linear differential equation in (7) renders two types of solutions - an homogeneous and a particular (or 
extremities and central solutions, respectively, as originally coined by Giavotto et al. [23]) - corresponding to two different physical phenomena. For a relatively long and slender beam, the homogeneous solution is associated with the deformations at the ends or extremities of the beam. The particular solution, on the other hand, will yield the displacement field at the central part of the beam where end effects become negligible. The latter will be used next to derive the stiffness properties of beam sections and consequently construct finite elements suitable for the analysis of the global behavior of relatively long and slender structures.

Before proceeding with the derivation note that the displacement definition in (5) is six times redundant. The warping displacements, $\mathbf{u}$, are able to replicate the six rigid body motions already included in $\psi$ (cf. (6)). The following set of constraint equations is therefore included (cf. Blasques [26])

$$
\left[\begin{array}{cc}
\mathbf{D}^{T} & \mathbf{0} \\
\mathbf{0} & \mathbf{D}^{T}
\end{array}\right]\left[\begin{array}{c}
\mathbf{u} \\
\frac{\partial \mathbf{u}}{\partial z}
\end{array}\right]=\left[\begin{array}{l}
\mathbf{0} \\
\mathbf{0}
\end{array}\right], \text { where } \mathbf{D}=\left[\begin{array}{ccc}
\mathbf{I}_{3} & \ldots & \mathbf{I}_{3} \\
\mathbf{n}_{1} & \ldots & \mathbf{n}_{n_{n}}
\end{array}\right]^{T}
$$

where $\mathbf{I}_{3}$ is the $3 \times 3$ identity matrix, and $\mathbf{n}_{n}$ is obtained from (1) using the nodal coordinates of node $n$. These constraints ensure that the warping displacements do not contribute to the rigid-body displacements of the cross section.

After some manipulation, the resulting equilibrium equations for a section in the central part of the beam are defined in matrix form as

$$
\mathbf{K w}=\mathbf{f} \Leftrightarrow\left[\begin{array}{cc}
\mathbf{K}_{11} & \mathbf{K}_{12} \\
\mathbf{0} & \mathbf{K}_{11}
\end{array}\right]\left[\begin{array}{l}
\mathbf{w}_{1} \\
\mathbf{w}_{2}
\end{array}\right]=\left[\begin{array}{l}
\mathbf{f}_{1} \\
\mathbf{f}_{2}
\end{array}\right]
$$

where

$$
\mathbf{K}_{11}=\left[\begin{array}{ccc}
\mathbf{E} & \mathbf{R} & \mathbf{D} \\
\mathbf{R}^{T} & \mathbf{A} & \mathbf{0} \\
\mathbf{D}^{T} & \mathbf{0} & \mathbf{0}
\end{array}\right], \mathbf{K}_{12}=\left[\begin{array}{ccc}
\left(\mathbf{C}^{T}-\mathbf{C}\right) & -\mathbf{L} & \mathbf{0} \\
\mathbf{L}^{T} & \mathbf{0} & \mathbf{0} \\
\mathbf{0} & \mathbf{0} & \mathbf{0}
\end{array}\right](10)
$$

and, $\mathbf{w}_{1}=\left[\begin{array}{lll}\mathbf{u}^{T} & \psi^{T} & \lambda_{1}^{T}\end{array}\right]^{T}, \mathbf{w}_{2}=\left[\partial \mathbf{u}^{T} / \partial z \partial \boldsymbol{\psi}^{T} / \partial z \lambda_{2}^{T}\right]^{T}, \mathbf{f}_{1}=$ $\left[\begin{array}{lll}\mathbf{0}^{T} & \boldsymbol{\theta}^{T} & \mathbf{0}^{T}\end{array}\right]^{T}$, and $\mathbf{f}_{2}=\left[\mathbf{0}^{T}\left(\mathbf{T}_{r}^{T} \boldsymbol{\theta}\right)^{T} \boldsymbol{0}^{T}\right]^{T}$. The vectors of Lagrange multipliers $\lambda_{1}$, and $\lambda_{2}$ refer to the first and second set of constraint equations, respectively. The set of equations above will yield the warping displacements and strains, $\mathbf{u}$ and $\psi$ respectively, for given internal section forces $\boldsymbol{\theta}$.

\subsubsection{Cross section stiffness properties}

A procedure is presented next for the derivation of the cross section compliance matrix $\mathbf{F}_{s}$ based on the cross section equilibrium equations derived in the previous section (cf. Giavotto et al. in [23]).

Consider the case where the set of equations (9) is solved for six different right-hand sides each corresponding to setting one of the entries of $\boldsymbol{\theta}$ to unity and the remaining to zero. This procedure can be realized by replacing the cross section load vector $\boldsymbol{\theta}$ by the $6 \times 6$ identity matrix $\mathbf{I}_{6}$ and solving the following set of equations

$$
\mathbf{K W}=\mathbf{F} \Leftrightarrow\left[\begin{array}{cc}
\mathbf{K}_{11} & \mathbf{K}_{12} \\
\mathbf{0} & \mathbf{K}_{11}
\end{array}\right]\left[\begin{array}{l}
\mathbf{W}_{1} \\
\mathbf{W}_{2}
\end{array}\right]=\left[\begin{array}{l}
\mathbf{F}_{1} \\
\mathbf{F}_{2}
\end{array}\right]
$$

where $\mathbf{W}_{1}=\left[\begin{array}{lll}\mathbf{U}^{T} & \boldsymbol{\Psi}^{T} & \boldsymbol{\Lambda}_{1}^{T}\end{array}\right]^{T}, \mathbf{W}_{2}=\left[\partial \mathbf{U}^{T} / \partial z \partial \boldsymbol{\Psi}^{T} / \partial z \boldsymbol{\Lambda}_{2}^{T}\right]^{T}$, $\mathbf{F}_{1}=\left[\begin{array}{lll}\mathbf{0}^{T} & \mathbf{I}_{6} & \mathbf{0}^{T}\end{array}\right]^{T}$, and $\mathbf{F}_{2}=\left[\begin{array}{lll}\mathbf{0}^{T} & \mathbf{T}_{r} & \mathbf{0}^{T}\end{array}\right]^{T}$. The resulting solution matrices $\mathbf{U}, \partial \mathbf{U} / \partial z, \boldsymbol{\Psi}$ and $\partial \boldsymbol{\Psi} / \partial z$ have six columns corresponding to each of the right-hand sides. The solutions vectors $\mathbf{w}_{1}$ and $\mathbf{w}_{2}$ can be retrieved for any $\boldsymbol{\theta}$ through $\mathbf{w}_{1}=\mathbf{W}_{1} \boldsymbol{\theta}$ and $\mathbf{w}_{2}=\mathbf{W}_{2} \boldsymbol{\theta}$, respectively.

The balance between the complimentary form of the cross section internal elastic energy and the internal virtual work or the elastic strain energy yields

$$
\delta \boldsymbol{\theta}^{T} \mathbf{F}_{s} \boldsymbol{\theta}=\delta \boldsymbol{\theta}^{T} \mathbf{W}^{T}\left[\begin{array}{ll}
\mathbf{G}_{11} & \mathbf{G}_{12} \\
\mathbf{G}_{12}^{T} & \mathbf{G}_{22}
\end{array}\right] \mathbf{W} \boldsymbol{\theta}=\delta \boldsymbol{\theta}^{T} \mathbf{W}^{T} \mathbf{G W} \boldsymbol{\theta}(12)
$$

where

$\mathbf{G}_{11}=\left[\begin{array}{ccc}\mathbf{E} & \mathbf{R} & \mathbf{0} \\ \mathbf{R}^{T} & \mathbf{A} & \mathbf{0} \\ \mathbf{0} & \mathbf{0} & \mathbf{0}\end{array}\right], \mathbf{G}_{12}=\left[\begin{array}{ccc}\mathbf{C} & \mathbf{L} & \mathbf{0} \\ \mathbf{L}^{T} & \mathbf{0} & \mathbf{0} \\ \mathbf{0} & \mathbf{0} & \mathbf{0}\end{array}\right], \mathbf{G}_{22}=\left[\begin{array}{ccc}\mathbf{M} & \mathbf{0} & \mathbf{0} \\ \mathbf{0} & \mathbf{0} & \mathbf{0} \\ \mathbf{0} & \mathbf{0} & \mathbf{0}\end{array}\right]$

The expression for the cross section compliance matrix is readily obtained from (12) as

$$
\mathbf{F}_{s}=\mathbf{W}^{T} \mathbf{G W}
$$

This concludes the derivation of the cross section stiffness matrix. In practice the procedure for the evaluation of $\mathbf{F}_{s}$ consists of first assembling the matrices in (8) using standard finite element techniques. The next step consists of assembling the matrices $\mathbf{K}_{11}$ and $\mathbf{K}_{12}$ in (10), and finding the solutions to (11). Finally, having assembled matrix $\mathbf{G}$, the cross section compliance matrix $\mathbf{F}_{s}$ is computed by replacing the solutions obtained into (13).

\subsubsection{Shear center position}

The shear and elastic center positions are determined based on the entries of the cross section compliance matrix (cf. Hodges [19]). The shear center, $\mathbf{s}_{c}=\left(x_{s}, y_{s}\right)$, of a given cross section is defined such that a transverse load applied at this point will not induce a torsional moment. It is given as a function of the cross section entries and defined as

$$
x_{s}=-\frac{F_{s, 62}+F_{s, 64}(L-z)}{F_{s, 66}}, \quad y_{s}=\frac{F_{s, 61}+F_{s, 65}(L-z)}{F_{s, 66}}(14)
$$

where $F_{s, i j}$ is the entry $(i, j)$ of the compliance matrix $\mathbf{F}_{s}$, and $L$ is the beam length.

\subsection{Beam finite element model}

The last step in the construction of the structural model entails the generation of the beam finite element matrices. The finite element form of the beam equilibrium equations is given by (see, e.g., Bathe [27])

$$
\widehat{\mathbf{K u}}_{l}=\widehat{\mathbf{f}}_{l}
$$

where $l=1, \ldots, n_{l}$, indicates the load case number from the $n_{l}$ load cases considered. The displacement vector $\widehat{\mathbf{u}}_{l}(x, y, z)$ holds 
the beam translational and rotational nodal degrees of freedom. The global beam finite element stiffness matrix $\widehat{\mathbf{K}}$ is defined as

$$
\widehat{\mathbf{K}}=\sum_{b=1}^{n_{b}} \widehat{\mathbf{K}}_{b}=\sum_{b=1}^{n_{b}} \int_{0}^{L_{b}} \widehat{\mathbf{B}}_{b}^{T} \mathbf{K}_{s} \widehat{\mathbf{B}}_{b} \mathrm{dz}
$$

where $\widehat{\mathbf{K}}_{b}$ is the beam finite element stiffness matrix for element $b, n_{b}$ is the number of beam elements in the finite element assembly, and $L_{b}$ is the length of element $b$. The beam strain-displacement matrix for element $b$ is defined from (6) as $\widehat{\mathbf{B}}_{b}=\left(\mathbf{T}_{r}+\partial / \partial z\right) \widehat{\mathbf{N}}_{b}(z)$ where $\widehat{\mathbf{N}}_{b}(z)$ is the matrix of shape functions. The global load vector $\widehat{\mathbf{f}}$ for load case $l$, is defined as

$$
\widehat{\mathbf{f}_{l}}=\sum_{b=1}^{n_{b}} \int_{0}^{L_{b}} \widehat{\mathbf{N}}^{T} \mathbf{f}_{b, l}^{s} \mathrm{dz}
$$

where loads $\mathbf{f}_{b, l}^{s}$ is the load components at element $b$. In the expressions above the sum refers to the typical finite element assembly procedure. The solution to the set of linear equations in (15) yields the nodal displacements and rotations $\widehat{\mathbf{u}}_{l}$ for a given beam finite element assembly subject to the loads $\widehat{\mathbf{f}}_{l}$.

\section{Formulation of the optimal design problem}

The formulation of the minimum compliance problem with constraints on weight and positions of shear and mass center is presented next. The aim is to identify the optimal distribution of a predefined number of candidate materials within a given cross section. The devised methodology should yield a solution where only one of the candidate materials has been chosen at each point of the design domain. In order to avoid the complications associated with the solution of discrete problems a relaxation has been suggested where the continuous design variables are allowed to vary between zero and one. Intermediate values are penalized by a material interpolation scheme thus forcing the design variables to converge to a discrete valued solution.

\subsection{Parameterization and penalization}

A SIMP-like material interpolation scheme (see Bendsøe and Kikuchi [28], Rozvany and Zhou [29]) is employed which can accommodate any number of anisotropic candidate materials. The $6 \times 6$ material constitutive matrix $\mathbf{Q}_{e}$ at element $e$ is assumed to be constant within each element and is defined as

$$
\mathbf{Q}_{e}(\boldsymbol{\rho})=\sum_{m=1}^{n_{c}} \widetilde{\rho}_{e m}^{p}(\boldsymbol{\rho}) \overline{\mathbf{Q}}_{m} \quad, \quad \forall e=1, \ldots, n_{e}
$$

where $n_{c}$ is the number of candidate materials and $n_{e}$ the number of elements in the cross section finite element mesh. The design variables $\rho=\left\{\rho_{e m} \in \mathbb{R} \mid e \in\left\{1, \ldots, n_{e}\right\}, m \in\left\{1, \ldots, n_{c}\right\}\right\}$ represent the volume fractions of each of the candidate materials $m$ represented by the constitutive matrix $\overline{\mathbf{Q}}_{m}$, at element $e$. The design variables are assumed to vary continuously between given bounds, i.e., $0 \leq \rho_{e m} \leq 1, \forall e=1, \ldots, n_{e}, \forall m=1, \ldots, n_{c}$.
The list of candidate materials is defined a priori and may include any type of anisotropic material as well as the same material aligned in different directions. The determination of the filtered volume fractions $\widetilde{\rho}_{e m}(\rho)$ is discussed in Section 3.2. The parameter $p \geq 1$ is a penalty term whose role will be discussed in further detail in the next section.

The element density $\varrho_{e}$ is given by

$$
\varrho_{e}(\boldsymbol{\rho})=\sum_{m=1}^{n_{c}} \widetilde{\rho}_{e m}(\boldsymbol{\rho}) \bar{\varrho}_{m} \quad, \forall e=1, \ldots, n_{e}
$$

where $\bar{\varrho}_{m}$ is the density of candidate material $m$.

Finally, the following linear equality constraints

$$
\sum_{m=1}^{n_{c}} \widetilde{\rho}_{e m}=1 \quad, \quad \forall e=1, \ldots, n_{e}
$$

are included in the problem formulation to ensure that the sum of the volume fractions of the candidate materials at each element or patch add to unity.

The design variables $\rho$ enter the structural model through the material constitutive matrices $\mathbf{Q}_{e}=\mathbf{Q}_{e}(\boldsymbol{\rho})$ as part of the definition of the finite element matrices in (8). Hence, $\mathbf{A}=\mathbf{A}(\boldsymbol{\rho})$, $\mathbf{M}=\mathbf{M}(\rho), \mathbf{C}=\mathbf{C}(\rho), \mathbf{E}=\mathbf{E}(\rho), \mathbf{R}=\mathbf{R}(\rho)$, and $\mathbf{L}=\mathbf{L}(\rho)$. Consequently, $\mathbf{F}_{s}=\mathbf{F}_{s}(\boldsymbol{\rho})$ at the cross section level and finally, $\widehat{\mathbf{K}}=\widehat{\mathbf{K}}(\boldsymbol{\rho})$ at the beam finite element level.

The parameterization presented before (assuming $p=1$ ) is prone to generate optimal designs where the solution is not discrete, i.e., where several candidate materials are combined in the same element. The SIMP penalization approach has been extensively used in topology optimization problems (see Bendsøe and Sigmund [1]) and is realized by controlling the penalty term $p \geq 1$ in (17). Increasing the value of $p$ corresponds to increasing the contrast between the different candidates and consequently in the penalization of intermediate densities.

The penalized problem is in general non-convex and may have a large number of local minima. Continuation methods have been suggested in this context as a way to increase the possibility of obtaining a good design (see Sigmund and Petersson [6], Borrvall and Petersson [30], and Hvejsel et al. [31]). In practice the problem is initially solved for $p=1$ corresponding to the case where no penalization is imposed. Subsequently, the penalty is increased and the found design of the former optimal design problem is used as the starting point of the new problem. As $p$ is increased the variables are forced to their bounds. This process is repeated until an increase in $p$ results in a negligible variation of the design variable values.

\subsection{Filtering scheme}

Typical issues in density based topology optimization include, among other, the appearance of checkerboards and meshdependency (Sigmund and Petersson [6]). Several regularization techniques have been proposed which introduce, in some way or another, a length scale on the optimal solution which eliminates these effects (see Sigmund and Petersson [6], Bruns 
gmund [32]). An extenand Tortorelli [8], Bourdin [7], and Sigmund [32]). An extenfor multi-material topology optimization problems is suggested here.

The set of elements surrounding element $e$ within a given radius $f_{r}$ is defined by the set $S_{e}$ such that, $S_{e}=$ $\left\{\tilde{e} \in\left\{1, \ldots, n_{e}\right\} \mid\left\|\mathbf{x}_{\tilde{e}}-\mathbf{x}_{e}\right\|_{2} \leq f_{r}\right\}$ where $\mathbf{x}_{\tilde{e}}$ and $\mathbf{x}_{e}$ are the coordinates of the centroid of element $\tilde{e}$ and $e$, respectively. The volume fraction of material $m$ at element $e$ is $\widetilde{\rho}_{e m}=\widetilde{\rho}_{e m}\left(\rho_{\tilde{e} m}\right), \forall \tilde{e} \in$ $S_{e}$. The filtered density for material $m$ at element $e$ is hence

$$
\widetilde{\rho}_{e m}=\frac{\sum_{\tilde{e} \in S_{e}} w\left(\mathbf{x}_{\tilde{e}}\right) v_{\tilde{e}} \rho_{\tilde{e} m}}{\sum_{\tilde{e} \in S_{e}} w\left(\mathbf{x}_{\tilde{e}}\right) v_{\tilde{e}}}
$$

The weighting function $w\left(x_{e}\right)$ is given by the function $w\left(\mathbf{x}_{\tilde{e}}\right)=$ $f_{r}-\left\|\mathbf{x}_{\tilde{e}}-\mathbf{x}_{e}\right\|_{2}$ as suggested by Bruns and Tortorelli [8] and Bourdin [7].

\subsection{Problem formulation}

The structural compliance of load case $l, c_{l}(\boldsymbol{\rho})=\widehat{\mathbf{f}}_{l}^{T} \widehat{\mathbf{u}}_{l}(\boldsymbol{\rho})$, is a measure of the stiffness of the beam defined as the work performed by the external loads. It is assumed herein that $\widehat{\mathbf{K}}(\rho)$ is positive definite for all $\rho$ within the specified bounds $0 \leq \rho_{e m} \leq 1, \forall e=1, \ldots, n_{e}, \forall m=1, \ldots, n_{c}$. Hence, it is possible to define the function $\widehat{\mathbf{u}}_{k}(\boldsymbol{\rho})=\widehat{\mathbf{K}}^{-1}(\boldsymbol{\rho}) \widehat{\mathbf{f}}_{k}$ such that $c_{l}(\boldsymbol{\rho})=\widehat{\mathbf{f}}_{l}^{T} \widehat{\mathbf{K}}^{-1}(\boldsymbol{\rho}) \widehat{\mathbf{f}}_{l}$. The weighted average of the compliances $C(\boldsymbol{\rho})$, for a given set of load cases is defined as

$$
C(\boldsymbol{\rho})=\sum_{l=1}^{n_{l}} \alpha_{l} c_{l}(\boldsymbol{\rho})=\sum_{l=1}^{n_{l}} \alpha_{l} \widehat{\mathbf{f}}_{l}^{T} \widehat{\mathbf{K}}^{-1}(\boldsymbol{\rho}) \widehat{\mathbf{f}}_{l}
$$

where $\alpha_{l} \geq 0$ is the given weight attributed to load case $l$. The formulation of the optimal design problem (P1) is then

$$
\begin{array}{cl}
\underset{\boldsymbol{\rho} \in \mathbb{R}^{n_{e} \times n_{c}}}{\operatorname{minimize}} & C(\boldsymbol{\rho}) \\
\text { subject to } & w(\boldsymbol{\rho}) \leq \bar{w} \\
& \mathbf{s}_{c}(\boldsymbol{\rho}) \leq \overline{\mathbf{s}} \\
& \mathbf{m}_{c}(\boldsymbol{\rho}) \leq \overline{\mathbf{m}} \\
& \sum_{m=1}^{n_{c}} \widetilde{\rho}_{e m}(\boldsymbol{\rho})=1, \forall e=1, \ldots, n_{e} \\
& 0 \leq \rho_{e m} \leq 1, \forall e=1, \ldots, n_{e}, \forall m=1, \ldots, n_{c}
\end{array}
$$

where $w(\boldsymbol{\rho})$ is the total beam weight. The parameters $\bar{w}, \overline{\mathbf{s}}$ and $\overline{\mathbf{m}}$ are the constraint values for the weight and, shear and mass center positions, respectively.

Finally, removing the shear and mass center position constraints from problem formulation (P1) will yield the formulation for the minimum compliance with weight constraints problem (P2).

\section{Sensitivity analysis}

The gradients (or sensitivities) of the beam compliance and cross section stiffness matrix are presented here. The sensitivities of the shear center position are obtained through differentiation of (14).

\subsection{Compliance}

The gradient of the compliance is derived in two steps. The first step consists of determining the partial derivative of the beam stiffness matrix, $\widehat{\mathbf{K}}(\boldsymbol{\rho})$. The second step consists of the determining an expression for the partial derivative of the cross section stiffness matrix, $\mathbf{K}_{s}(\boldsymbol{\rho})$.

Since the loads are assumed to be design independent, the partial derivative of $C(\boldsymbol{\rho})$ with respect to design variables $\rho_{e m}$ is

$$
\frac{\partial C(\boldsymbol{\rho})}{\partial \rho_{e m}}=\sum_{l=1}^{n_{l}} \alpha_{l} \frac{\partial c_{l}(\boldsymbol{\rho})}{\partial \rho_{e m}}=\sum_{l=1}^{n_{l}} \alpha_{l} \widehat{\mathbf{f}}_{l} \frac{\partial \widehat{\mathbf{u}}_{l}(\boldsymbol{\rho})}{\partial \rho_{e m}}=\widehat{\mathbf{f}}_{l}^{T} \frac{\partial \widehat{\mathbf{K}}(\boldsymbol{\rho})^{-1}}{\partial \rho_{e m}} \widehat{\mathbf{f}}_{l}
$$

In this case the following holds (see, e.g., Bendsøe and Sigmund [1])

$$
\frac{\partial c_{l}(\boldsymbol{\rho})}{\partial \rho_{e m}}=-\widehat{\mathbf{u}}_{l}(\boldsymbol{\rho})^{T} \frac{\partial \widehat{\mathbf{K}}(\rho)}{\partial \rho_{e m}} \widehat{\mathbf{u}}_{l}(\boldsymbol{\rho})
$$

The partial derivative of the global beam stiffness matrix $\widehat{\mathbf{K}}$ is obtained from the differentiation of (16) and is defined as

$$
\frac{\partial \widehat{\mathbf{K}}(\boldsymbol{\rho})}{\partial \rho_{e m}}=\sum_{b=1}^{n_{b}} \int_{0}^{L_{b}} \widehat{\mathbf{B}}_{b}^{T} \frac{\partial \mathbf{K}_{s}(\boldsymbol{\rho})}{\partial \rho_{e m}} \widehat{\mathbf{B}}_{b} \mathrm{dz}
$$

Computing the gradient of the beam compliance reduced to the evaluation of the partial derivatives of the cross section stiffness matrix $\mathbf{K}_{s}(\boldsymbol{\rho})$.

\subsubsection{Cross section stiffness matrix}

The derivative of the cross section stiffness matrix $\mathbf{K}_{s}$ with respect to the design variable $\rho_{e m}$ is

$$
\frac{\partial \mathbf{K}_{s}(\boldsymbol{\rho})}{\partial \rho_{e m}}=\frac{\partial \mathbf{F}_{s}^{-1}(\boldsymbol{\rho})}{\partial \rho_{e m}}=-\mathbf{K}_{s}(\boldsymbol{\rho}) \frac{\partial \mathbf{F}_{s}(\boldsymbol{\rho})}{\partial \rho_{e m}} \mathbf{K}_{s}(\boldsymbol{\rho})
$$

where $\mathbf{F}_{s}^{-1}=\mathbf{K}_{s}$. From (13), the cross section compliance matrix is defined as $\mathbf{F}_{s}=\mathbf{W}^{T}(\boldsymbol{\rho}) \mathbf{G}(\boldsymbol{\rho}) \mathbf{W}(\boldsymbol{\rho})$ where the solution vectors in $\mathbf{W}(\boldsymbol{\rho})$ are computed as $\mathbf{W}(\boldsymbol{\rho})=\mathbf{K}^{-1}(\boldsymbol{\rho}) \mathbf{F}$ from (11). Employing the chain rule yields

$$
\begin{aligned}
\frac{\partial \mathbf{F}_{s}(\boldsymbol{\rho})}{\partial \rho_{e m}}= & -\mathbf{W}^{T}(\boldsymbol{\rho}) \frac{\partial \mathbf{K}^{T}(\boldsymbol{\rho})}{\partial \rho_{e m}} \mathbf{V}(\boldsymbol{\rho})+\mathbf{W}^{T}(\boldsymbol{\rho}) \frac{\partial \mathbf{G}(\boldsymbol{\rho})}{\partial \rho_{e m}} \mathbf{W}(\boldsymbol{\rho}) \\
& -\mathbf{V}^{T}(\boldsymbol{\rho}) \frac{\partial \mathbf{K}(\boldsymbol{\rho})}{\partial \rho_{e m}} \mathbf{W}(\boldsymbol{\rho})
\end{aligned}
$$

where $\mathbf{V}(\rho)$ is obtained from the solution to

$$
\mathbf{K}^{T}(\rho) \mathbf{V}(\rho)=\mathbf{G}(\rho) \mathbf{W}(\rho)
$$

The system of linear equations above is solved only once regardless of the number of design variables. The gradient of the 
$A$ C

cross section stiffness matrix is obtained by inserting the result from (21) into (20). Finally, replacing (20) in (19) and the corresponding result in (18) yields the gradient of the beam compliance for load case $l$. The reader is referred to Blasques [26] for further details on the derivation of the cross section stiffness matrix.

\section{Numerical experiments}

In this section we present a set of numerical experiments which illustrate the behavior of the devised methodology when applied to the optimal design of laminated composite beams. The setup of the experiments, namely the beam geometries, material properties, and load cases is presented first. Details regarding the organization and visualization of the results are discussed next. Finally, all numerical results are presented and discussed.

\subsection{Beam geometries and material properties}

Three different cross section geometries have been considered - a square, an L-shape, and a wing profile from a section of a wind turbine blade. The dimensions and finite element discretizations of the cross sections are presented in Figure 2. The cross section finite element discretization is based on two dimensional four node isoparametric finite elements (see Bathe [27]).

For all cases, the length of the cantilever beams is 20 meter and the beam finite element model is composed of 32 three node quadratic beam finite elements. The beam finite element discretization consists of three node quadratic beam finite elements. In Figure 2 the position of the beam node with respect to the cross section geometry is represented by a square marker.

The material properties are specified at each element of the cross section finite element discretization. The candidate materials may be isotropic, anisotropic, or even the same anisotropic material oriented in different directions. In the numerical experiments, two material types have been considered - one orthotropic laminate and one isotropic material. The orthotropic laminate corresponds to a type of E-Glass reinforced Epoxy (cf. Peters [33]) whose properties are $E_{11}=480 \mathrm{Gpa}, E_{22}=$ $E_{33}=120 \mathrm{Gpa}, G_{12}=G_{13}=60 \mathrm{GPa}, G_{23}=50, v_{12}=v_{13}=0.19$, $v_{23}=0.26$, and $\bar{\varrho}=1780 \mathrm{~kg} / \mathrm{m}^{3}$. The isotropic material corresponds to a type of polyvinyl chloride (PVC) core material (cf. DIAB H100 [34]) typically used in sandwich structures of wind turbine blades. The properties of the isotropic material are $E=0.130 \mathrm{Gpa}, G=0.035 \mathrm{GPa}, v=0.35$, and $\bar{\varrho}=100 \mathrm{~kg} / \mathrm{m}^{3}$.

\subsubsection{Generating candidates}

A candidate material constitutive matrix $\overline{\mathbf{Q}}_{j}$ is generated in the following manner. The material constitutive matrices, $\mathbf{Q}_{m}$, for each material $m$ are based on the material properties specified in Section 5.1. This will initially yield two candidates the orthotropic material and the isotropic material, respectively. The remaining candidates are generated by orienting the material constitutive matrix $\mathbf{Q}_{m}$ of the orthotropic material in different directions. Hence, the material constitutive matrix of candidate $j$ is obtained from the rotation of the material constitutive matrix $\mathbf{Q}_{m}$ as $\overline{\mathbf{Q}}_{j}=\mathbf{R}_{t} \mathbf{Q}_{m} \mathbf{R}_{t}^{T}$ where $\mathbf{R}_{t}$ is the three dimensional transformation matrix for a rotation about a given axis. This is a convenient approach in the design of laminated composite structures. A laminate consists of different layers of fibers aligned in different directions. The layers will be stacked in different directions defined by the different fiber plane orientations. In this case, a different matrix $\overline{\mathbf{Q}}_{j}$ will be associated with each different combination of fiber and fiber plane orientation generated from the same matrix $\mathbf{Q}_{m}$. The procedure is illustrated in Figure 3. First, the fiber plane orientation is defined by the rotation $\alpha_{p}$. Then the fibers are rotated in their own stacking plane by $\alpha_{f}$ and the final fiber orientation is obtained.

In total eight orthotropic candidate materials have been considered during the numerical experiments. These materials correspond to four different in-plane fiber orientations $\left(0^{\circ}, 45^{\circ}\right.$, $-45^{\circ}$, and $\left.90^{\circ}\right)$ stacked in two different fiber plane orientations $\left(0^{\circ}\right.$ and $90^{\circ}$ or horizontal and vertical, respectively). The list of candidate materials is described in Figure 5 where the resulting spatial orientation of the fibers for each of the orthotropic candidates are listed.

\subsection{Load cases}

All loads are applied as distributed pressure loads along the beam length. The direction of the loads is according to the cross section coordinate system shown in Figure 1. The load cases for the square and the L-shape cross sections are presented in Table 1.

The beam with the wing profile cross section is subjected to 15 static load cases. The load cases are defined based on the aerodynamic loading generated by an airfoil of the same shape exposed to a constant incoming wind speed of $W_{s}=20 \mathrm{~m} / \mathrm{s}$ at 15 different angles of attack. For each angle of attack the aerodynamic loads are computed as $L_{a}=\frac{1}{2} \varrho_{a} W_{s}^{2} c C_{L}, D_{a}=$ $\frac{1}{2} \varrho_{a} W_{s}^{2} c C_{D}, M_{a}=\frac{1}{2} \varrho_{a} W_{s}^{2} c^{2} C_{M}$ where $L_{a}, D_{a}$, and $M_{a}$, are the aerodynamic lift, drag, and moment, respectively. The aerodynamic lift, drag and moment coefficients, $C_{L}, C_{D}$, and $C_{M}$, respectively, are obtained from the experimental data in Ramsay et al. [35]. The experimental data is presented in Figure 4(a) for several values of angle of attack $\alpha_{w}$. The air density is assumed to be $\varrho_{a}=1.2041 \mathrm{~kg} / \mathrm{m}^{3}$. The magnitude of lift and drag is defined with respect to the wind direction as described in Figure 4(b). Its components with respect to the cross section coordinate system are computed to define the corresponding resultant load vector $R_{a}$. The magnitude and orientation of the aerodynamic lift and drag loads, and corresponding resultant vector, for each of the 15 load cases are also presented in Figure 4(b). Note that the aerodynamic moment is not depicted in these figures.

The idealized wind turbine blade is represented by a cantilever beam of constant cross section along the length. The static pressure loads are also assumed to be constant along the length of the beam. The beam node or the point of application of the loads is coincident with the aerodynamic center positioned at a distance equal to a quarter of the chord from the leading edge (see Figure 2). 


\subsection{Optimization strategy}

The optimization strategy consists of first solving the unpenalized problem, i.e., assuming $p_{1}=1$ in the material interpolation scheme in (17). The penalty parameter is then gradually increased such that at step $i+1$ the penalty value is $p_{i+1}=p_{i}+\Delta p$, where $\Delta p \geq 0$. Each problem is solved until some optimality criteria is satisfied or the maximum number iterations is met. In SNOPT, the major optimality tolerance and maximum number of major iterations (number of solved quadratic sub-problems) at each step of the continuation method is set to $1 \times 10^{-5}$ and 500, respectively. The remaining parameters in SNOPT are set to the default values. In the numerical experiments using the square and L-shape cross sections a $\Delta p=0.1$ has been used. In the case of the wing profile a smaller step length of $\Delta p=0.075$ is used. For all cases the final penalty value is such that $p \geq 3$ (see Table 3). Finally, the starting point for all cases is such that the same volume fraction is given to all materials.

\subsection{Presentation of the results}

The optimal distribution of candidate materials is presented using two figures. The two figures show the fiber and fiber plane orientations at each element, respectively. In both cases the orientation is marked with a line on the element. The orientation of these lines define the spatial orientation of the fibers of the laminated orthotropic material at each element as described in Figure 5. The thickness and darkness of the line is weighted by the value of the filtered design variable at the corresponding element. It is consequently possible to visualize the effect of the density filter in the areas of transition between different materials. Finally, the element is white in case the material is isotropic.

Note that for all cases, the presented designs correspond to the unpenalized filtered volume fractions. The use of the filtered values is motivated by the fact that these represent the actual material volume fractions multiplying each of the candidates.

\subsection{Results}

All numerical experiments are listed in Table 2 as a combination between the different cross sections, load cases, and problem formulations. Details regarding the problem size, number of design variables, step length and maximum penalty size in the continuation method, and the chosen constraint values are presented in Table 3. The same table also presents the resulting number of objective function evaluations, and objective function and constraint values for all the optimal design problems.

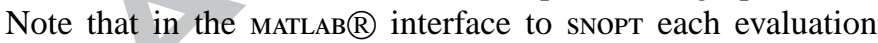
of the objective function and constraints entails necessarily an evaluation of its gradients.

The results obtained for the minimum compliance problem with weight constraints (P2) are presented first. The results for the minimum compliance problem with constraints on weight and shear and mass center positions (P1), are presented next. Finally, the devised methodology is applied in the optimal design of the structural lay-out of a wind turbine blade cross section.
5.5.1. Minimum compliance with weight constraints

The optimal design presented in Figure 6(a,d), correspond to the case where the beam is subjected to a distributed vertical load (case S1). The normal stresses induced by the bending moment dominate in the top and bottom regions away from the neutral axis. In the central part of the beam, close to the neutral axis, the normal stresses approach zero and the shear stresses dominate. The resulting topology is a variation of the typical I-beam or box-beam with the flanges resisting the normal stresses and the webs resisting the shear stresses. Regarding the fiber orientations, the laminates at the flanges orient at $0^{\circ} @ 90^{\circ}$ aligned along the length of the beam to resist the normal stresses. The laminate structure in the webs is composed of $45^{\circ} @ 90^{\circ}$ and $-45^{\circ} @ 90^{\circ}$. These patterns in terms of fiber and fiber plane orientations are common to most of the solutions where a transverse load dominates the load case. Note that the same pattern emerges in cases L1 and L3 in Figures $7(\mathrm{a}, \mathrm{d})$ and 7(b,e), respectively. The regions away from the neutral axis are composed of $0^{\circ} @ 0^{\circ}$ and $0^{\circ} @ 90^{\circ}$ laminates. The fiber plane orientation changes as the fibers go around a corner of the cross section. In these cases the areas dominated by shear also present the same type of laminate structure with two separate regions of $45^{\circ} @ 90^{\circ}$ and $-45^{\circ} @ 90^{\circ}$ for vertical webs, and $45^{\circ} @ 0^{\circ}$ and $-45^{\circ} @ 0^{\circ}$ for horizontal ones.

Note that for case $\mathrm{S} 1$ (see Figure $6(\mathrm{a}, \mathrm{d})$ ) the relative position of the shear center coincides with the beam node, or the point of application of the loads. Thus, an applied transverse force, irrespective of the direction, will not induce a torsional moment. In case L1 (see Figure 7(a,d)) the shear center position is aligned with the beam node along the direction of the applied transverse load (load case LC $2, \widehat{f_{x}}+\widehat{f_{y}}$ ). Hence, the specific load combination in LC2 will not induce a torsional moment, but the coupling will occur for a transverse load in any other direction.

The results obtained for the L-shape cross section subject to a torsional moment (case L2) is presented in Figures 7(b,e). As expected, the resulting optimal design is a closed cross section. This is due to the fact that for a closed cross section subjected to torsion the shear flow is constant through the thickness. In open cross sections the shear flow varies linearly through the thickness. Hence, the torsional stiffness is a function of the area for closed cross sections and of the thickness for open cross sections. Thus, closed cross sections are stiffer in torsion. An assumption of the Saint-Venant theory underlying the structural model used here is that the warping deformation is unconstrained. As such, for a beam subjected to a torsional moment, the normal stresses are nil and the cross section walls are subject to shear stresses only. The resulting optimal solution consists therefore only of laminates oriented at $45^{\circ} @ 0^{\circ},-45^{\circ} @ 0^{\circ}$, $45^{\circ} @ 90^{\circ}$, and $-45^{\circ} @ 90^{\circ}$. In this way the fibers align in the direction of the principal stresses. Moreover, a layered structure is visible which is in agreement with the common engineering intuition. Since both $45^{\circ}$ and $-45^{\circ}$ directions are equally stiff, a stacking of interchanging layers of $45^{\circ}$ and $-45^{\circ}$ increases stiffness and avoids any elastic anisotropic material couplings.

Contrarily to the result obtained for case $\mathrm{S} 1$, in the resulting designs for cases S2 and S3 (Figures 6(b,e) and 6(c,f), respec- 
tively) the shear center does not coincide with the point of application of the loads. The position of the shear center $\mathbf{s}_{c}$ is a function of the magnitude of the entries of the cross section compliance matrix $\mathbf{F}_{s}, F_{s, 61}$ and $F_{s, 62}$ for the shear-torsion couplings, and, $F_{s, 64}$ and $F_{s, 65}$ for the bending-torsion couplings (cf. (14) in Section 2.1). The magnitude of these entries depends on both the cross section topology and the elastic couplings stemming from material anisotropy. In the $S 2$ case (see Figure $6(b, e)$ ) it is visible that the optimal solution has exploited both effects. The topology of the cross section is such that there is an asymmetry with respect to the vertical axis. Moreover, existence of a layer of $-45^{\circ} @ 0^{\circ}$ in both top and bottom will result in nonzero shear- and bend-torsion coupling coefficients. Both these features contribute to the horizontal shift in the position of the shear center. The same mechanisms play an identical role in the optimal solution of case S3 (see Figure 6(c,f)). In summary, the beams of case S2 and S3 will present couplings between both shear and torsion and, bending and torsion. In these cases, and based on the position of the shear center, these couplings are such that the applied transverse force $-\widehat{f_{y}}$ in S 2 and, $\widehat{f}_{x}+\widehat{f_{y}}$ in $\mathrm{S} 3$ - will induce a torsional rotation of the cross section which opposes the applied torsional moment $\widehat{m}_{x}$.

\subsubsection{Minimum compliance problem with constraints on mass and positions of shear and mass centers.}

The S3 and L1 cases (see Figures 6(c,f) and 7(a,d) respectively) are revisited here but now the positions of the shear and mass center are constrained. The resulting optimal designs are presented in Figures 8 and 9, for case S4 and L4, respectively. The resulting values of the constraints are presented in Table 3 . In the S4 case the shear and mass center positions are constrained such that its position is coincident with the beam node, i.e., $x_{s}=0, y_{s}=0, x_{c}=0$, and $y_{c}=0$. The resulting design satisfies all constraints. Compared to the S3 case (see Figure $6(c, f)$, the orientation of the fibers is similar in the different regions. The thickness of the faces however has changed to satisfy the constraints in the mass center position. The resulting compliance for the $\mathrm{S} 4$ case is, as a consequence, slightly higher than that of case S3 (cf. the results in Table 3).

In the L4 case (see Figure 9) the shear and mass center are constrained such that $0 \leq x_{s} \leq 1,0 \leq y_{s} \leq 1,0 \leq x_{c} \leq 1$, and $0 \leq y_{c} \leq 1$. The resulting optimal design satisfies all the constraints. The resulting cross section topology fiber orientations are similar to the unconstrained case L1 (see Figure 7(a,d)). The material however has been redistributed to satisfy the constraint on the mass center position which is now placed lower than in the L1 case.

\subsubsection{Application to the structural design of the cross section of a wind turbine blade}

The same methodology is now applied in the design of the cross section of an idealized wind turbine blade. The blade is subject to 15 static load cases as described in Section 5.2.

For the minimum compliance problem with weight constraints (problem formulation (P2)) the ratio between the stiffness and density of the materials does not affect the results. This is not the case when the constraints on shear and mass center positions are included (problem formulation (P1)). Both the shear and mass center positions are functions of the relative difference between the stiffness and density of the candidate materials. In order to obtain realistic values a second isotropic material is included which scales the first isotropic materials stiffness and density by a factor of $1 \times 10^{-3}$. The aim is to include a "material" which mimics void as commonly done in topology optimization. Thus, in both the W1 and W2 cases ten candidate materials have been considered (eight orthotropic and two isotropic materials) instead of the nine considered in the previous cases.

Note that the aim here is to design the load carrying structure of the wind turbine blade. It is therefore assumed that the aerodynamic shell is non-structural and exists around the perimeter of the cross section shape outside the design domain.

The results are presented in Figures 10 and 11 for cases W1 and W2, respectively. In both cases only the void material (or second and weaker isotropic material) exists in both final optimal designs. Furthermore, both results suggest that the effect of the aerodynamic moment $M_{a}$ is small and that the distributed transverse forces $R_{a}$ are the dominating loads. Hence, the resulting optimal designs present patterns similar to the obtained for the square cross section under a transverse load (case S1, Figure $6(\mathrm{a}, \mathrm{d})$, respectively). The laminates in top and bottom form the flanges of the box beam resisting the normal stresses. The fibers in these regions are oriented along the length of the blade and stacked in an horizontal plane, i.e., the $0^{\circ} @ 0^{\circ}$ laminate. The webs or side faces of the box beam resist the shear stresses. The laminates here are mostly composed of fibers oriented at $45^{\circ}$ and $-45^{\circ}$ and stacked in a vertical plane, i.e., laminates $45^{\circ} @ 90^{\circ}$, and $-45^{\circ} @ 90^{\circ}$. The resulting optimal designs are similar to existing wind turbine blade designs where the load carrying structure consists of a box beam or spar, see, e.g., [36].

Usually, one of the most important design drivers for the structural design of laminated composite wind turbine blades made of E-glass is tower clearance, i.e., the blade may not hit the tower. Hence, in order to increase the cross section moment of inertia and maximize the blade stiffness it is obvious to make the laminate in the flanges thicker in the regions where the airfoil is thicker. However, following this procedure will typically result in a design where the mass center is positioned too far aft, away from the aerodynamic center. This will often lead to undesirable issues related with blade flutter (see, e.g., Hansen [16]). Hence, the optimal blade structural layout should present, among other, the best compromise between stiffness and relative position of aerodynamic and mass center.

These considerations have been incorporated in the W2 case (see Figure 11) where the shear and mass centers positions have been constrained. Hence, the distance from the mass center to the aerodynamic center is reduced to about half the distance obtained in $\mathrm{W} 1$, i.e., $x_{c} \leq 0.1$. In order to avoid strong couplings between the transverse (or flap wise) and torsional deformation, the shear center is constrained so that it remains within a given distance of the aerodynamic center, i.e., $x_{s} \geq-0.2$. The aim is to design the cross section structural lay-out and simultaneously account for the static and dynamic properties of the wind 
turbine blade. The resulting optimal design satisfies both constraints. The difference in the results from the $\mathrm{W} 1$ and $\mathrm{W} 2$ case can be interpreted as a shift towards the leading edge in the position of the box beam. However, note that the resulting optimal design W2 is more compliant than the optimal design from case W1.

\section{Conclusions and future research}

We have presented a beam finite element formulation for the analysis of anisotropic and inhomogeneous beams with arbitrary cross section geometries. The formulation builds on a finite element discretization of the cross section geometry making it possible to use standard density-based topology optimization techniques. We have also formulated a minimum compliance multi-material topology optimization problem with constraints on the weight and shear and mass center positions. The design variables represent the volume fractions of each of the members in a predefined list of candidate materials. An extension of the SIMP material interpolation scheme and density filtering has been presented which can accommodate any number of (anisotropic) materials.

Numerical experiments have been presented to illustrate the numerical behavior of the proposed framework. The cross section topology and material properties have been optimized for two different basic structures - a square and L-shape beam sections - subjected to different load cases. The applicability of the framework has also been demonstrated in the optimal design of the cross section of an idealized wind turbine blade. The results indicate that the proposed methodology is suitable for the optimization of cross section topology and material distribution in minimum compliance design of laminated composite beams with prescribed weight and, shear and mass center placement constraints.

The next step in the research includes optimal design of beams with varying cross section properties along the length, and the incorporation of frequency and aeroelastic constraints. The advantages of the beam finite element model will be evident in the latter case. The reduced size of the beam finite element matrices will allow for the efficient analysis of the global beam response. Consequently, non-linear phenomena like large displacements and coupled aeroelastic loads will be accounted for with minor computational effort. As a result it will be possible to truly address the realistic design of wind turbine blades within an optimal design framework.

\section{Acknowledgements}

The authors wish to thank Christian Frier Hvejsel at Aalborg University for fruitful discussions and suggestions. The computer resources were funded by the Danish Center for Scientific Computing (www.dcsc.dk) under the grant CPU-0107-07 Optimal design of composite structures.

1. Bendsøe MP, Sigmund O. Topology optimization: theory, methods, and applications. Springer Verlag; 2nd ed.; 2003. ISBN 3540429921.
2. Sigmund O, Torquato S. Design of materials with extreme thermal expansion using a three-phase topology optimization method. Journal of the Mechanics and Physics of Solids 1997;45(6):1037-1067.

3. Lund E, Stegmann J. On structural optimization of composite shell structures using a discrete constitutive parametrization. Wind Energy 2005; 8(1):109-124.

4. Stegmann J, Lund E. Discrete material optimization of general composite shell structures. International Journal for Numerical Methods in Engineering 2005;62(14):2009-2027.

5. Bends $\varnothing$ e MP, Sigmund O. Material interpolation schemes in topology optimization. Archive of Applied Mechanics 1999;69(9):635-654.

6. Sigmund O, Petersson J. Numerical instabilities in topology optimization: A survey on procedures dealing with checkerboards, mesh-dependencies and local minima. Structural Optimization 1998;16(1):68-75.

7. Bourdin B. Filters in topology optimization. International Journal for Numerical Methods in Engineering 2001;50(9):2143-2158.

8. Bruns T, Tortorelli D. Topology optimization of non-linear elastic structures and compliant mechanisms. Computer Methods in Applied Mechanics and Engineering 2001;190(26-27):3443-3459.

9. Blasques JP, Stolpe M. Maximum stiffness and minimum weight optimization of laminated composite beams using continuous fiber angles. Structural and Multidisciplinary Optimization 2010;43(4):573-588.

10. Li L, Volovoi V. Cross-sectional design of composite rotor blades. Journal of the American Helicopter Society 2008;129(3):2865.

11. Ganguli R, Chopra I. Aeroelastic optimization of a helicopter rotor with composite coupling. Journal of Aircraft 1995;32(6):1326-1334.

12. Murugan S, Ganguli R. Aeroelastic stability enhancement and vibration suppression in a composite helicopter rotor. Journal of aircraft 2005; (4):1013-1024.

13. Kim YY, Kim TS. Topology optimization of beam cross sections. International Journal of Solids and Structures 2000;37(36):477-493.

14. Kim TS, Kim YY. Multiobjective Topology Optimization of a Beam Under Torsion and Distortion. Journal of the American Institute of Aeronautics and Astronautics 2002;40(2):376-381.

15. Donoso A, Sigmund O. Topology optimization of multiple physics problems modelled by Poissons equation. Latin American Journal of Solids and Structures 2004;1:169-184.

16. Hansen M. Aeroelastic instability problems for wind turbines. Wind Energy 2007;10(6):551-577.

17. Jung S, Nagaraj V, Chopra I, Others . Assessment of composite rotor blade modeling techniques. Journal of the American Helicopter Society 1999;44(3): 188

18. Volovoi V, Hodges D, Cesnik C, Popescu B. Assessment of beam modeling methods for rotor blade applications. Mathematical and Computer Modelling 2001;33(10-11):1099-1112.

19. Hodges DH. Nonlinear composite beam theory; vol. 3. American Institute of Aeronautics and Astronautics; 2006.

20. Yu W, Hodges DH. Generalized Timoshenko theory of the variational asymptotic beam sectional analysis. Journal Of The American Helicopter Society 2005;50(1):46.

21. Berdichevsky V. On the energy of an elastic beam. Journal of Applied Mechanics 1981;45(4):518-529.

22. Volovoi VV, Hodges DH, Vam B. Validation of the variational asymptotic beam sectional analysis (VABS). AIAA Journal 2002;40(10):2105-2112.

23. Giavotto V, Borri M, Mantegazza P, Ghiringhelli G, Carmaschi V, Maffioli $\mathrm{G}$, et al. Anisotropic beam theory and applications?. Computers $\mathcal{E}$ Structures 1983;16(1-4):403-413.

24. Ghiringhelli G, Mantegazza P. Linear, straight and untwisted anisotropic beam section properties from solid finite elements. Composites Engineering 1994;4(12):1225-1239.

25. Ghiringhelli $\mathrm{G}$. On the thermal problem for composite beams using a finite element semi-discretisation. Composites Part B: Engineering 1997; 28(5-6):483-495.

26. Blasques JP. Optimal Design of laminated composite beams. Phd thesis; Technical University of Denmark; 2011.

27. Bathe KJ. Finite Element Procedures. Prentice Hall; 1996.

28. Bendsøe MP, Kikuchi N. Generating optimal topologies in structural design using a homogenization method. Computer Methods in Applied Mechanics and Engineering 1988;71:197-224.

29. Rozvany G. I. , Zhou M. The COC algorithm, Part I: Cross-section optimization or sizing. Computer Methods in Applied Mechanics and Engi- 
neering 1991;89:281-308.

30. T. B, J. P. Topology optimization using regularized intermediate density control. Computer Methods in Applied Mechanics and Engineering 2001; 190(37-38):4911-4928.

31. Hvejsel CF, Lund E. Material interpolation schemes for unified topology and multi-material optimization. Structural and Multidisciplinary Optimization 2011;43(1997):811-825.

32. Sigmund O. Morphology-based black and white filters for topology optimization. Structural and Multidisciplinary Optimization 2007;33(45):401-424.

33. Peters S. Handbook of composites. London: Chapman and Hall; 2nd ed.; 1998.

34. DIAB Group . Divinycell H100 Data Sheet. 2011. URL www. diabgroup. com.

35. Ramsay R, Hoffmann M, Gregorek G. Effects of Grit Roughness and Pitch Oscillations on the S809 Airfoil: Airfoil Performance Report. Tech. Rep.; The Ohio State University; Ohio; 1999.

36. Energy AP. Company focus. $2011 . \quad$ URL allpoints-energy.com/focus. 
Vitae

José Pedro Blasques completed his Ph.D. studies at the Technical University of Denmark with the thesis entitled Optimal design of laminated composite beams. He was a visiting researcher at the National Renewable Energy Laboratory (CO, USA) and at the University of Colorado (CO, USA). $\mathrm{He}$ is currently a Postdoc at DTU Wind Energy, Technical University of Denmark, working on structural optimization of wind turbine components. His research focuses on analysis and design optimization of laminated composite structures.

Mathias Stolpe obtained his PhD in Optimization and Systems Theory from the Royal Institute of Technology (KTH), Stockholm, Sweden in 2003. In the period 20032011 he was working as assistant/associate professor at DTU Mathematics, Technical University of Denmark (DTU). He currently holds a position as Senior Scientist at DTU Wind Energy. His main areas of research are theory and algorithms for global optimization in optimal design, in particular topology optimization.

\section{Funding}

The project was fully funded by the Technical University of Denmark which attributed a Ph.D. scholarship to the first author. 
Table 1: Load cases and direction and magnitude of the loads, for two of the beam geometries. The distributed transverse forces $\widehat{f_{x}}$ and $\widehat{f_{y}}$, and the torsional moment $\widehat{m}_{z}$ have been considered.

\begin{tabular}{cccc}
\hline \multirow{2}{*}{ Load cases } & \multirow{2}{*}{ Direction } & \multicolumn{2}{c}{ Magnitude } \\
\cline { 3 - 4 } & & Square & L-shape \\
\hline LC1 & $\widehat{f}_{y}$ & 0.3 & - \\
LC2 & $\widehat{f}_{x}+\widehat{f}_{y}$ & $0.2+0.3$ & $0.2+0.35$ \\
LC3 & $\widehat{m}_{z}$ & 1.5 & 1 \\
LC4 & $\widehat{f}_{y}+\widehat{m}_{z}$ & $0.3+1.5$ & - \\
LC5 & $\widehat{f}_{x}+\widehat{f}_{y}+\widehat{m}_{z}$ & $0.2+0.3+1.5$ & - \\
LC6 & $\widehat{f}_{x}, \widehat{f}_{y}$ & $0.2,0.3$ & $0.2,0.35$ \\
\hline
\end{tabular}

Table 2: Catalog of problems combining different cross sections (square, Lshape, and wing profile as described in Figure 2), load cases (cf. Table 1), and problem formulations. The minimum compliance problem with constraints on weight and positions of shear and mass center is denoted (P1), and the minimum compliance problem with weight constraints is denoted (P2).

\begin{tabular}{cccc}
\hline Ref. & $\begin{array}{c}\text { Cross } \\
\text { section }\end{array}$ & $\begin{array}{c}\text { Load } \\
\text { case }\end{array}$ & $\begin{array}{c}\text { Problem } \\
\text { formulation }\end{array}$ \\
\hline S1 & Square & LC1 & $(\mathrm{P} 2)$ \\
S2 & Square & LC4 & $(\mathrm{P} 2)$ \\
S3 & Square & LC5 & $(\mathrm{P} 2)$ \\
S4 & Square & LC6 & $(\mathrm{P} 1)$ \\
L1 & L-shape & LC2 & $(\mathrm{P} 2)$ \\
L2 & L-shape & LC3 & (P2) \\
L3 & L-shape & LC6 & (P2) \\
L4 & L-shape & LC2 & (P1) \\
W1 & Profile & Aerodynamic & (P2) \\
W2 & Profile & Aerodynamic & (P1) \\
\hline
\end{tabular}


Table 3: Details of numerical experiments and summary of results for all cases in Table 2. The first column indicates the number of degrees of freedom (cross section/beam) while the second column indicates the total number of design variables. The next two columns indicate the step size and maximum penalty ( $\Delta p$ and $p_{\max }$, respectively) associated with the continuation method. The next three columns indicate the values of the constraints on weight (defined by the ratio of orthotropic material), shear center and mass center positions $\left(\bar{w}, \overline{\mathbf{s}}_{c}=\left(\bar{x}_{s}, \bar{y}_{s}\right)\right.$, and $\overline{\mathbf{m}}_{c}=\left(\bar{x}_{c}, \bar{y}_{c}\right)$, respectively). The remaining columns refer to the number of objective function evaluations, the resulting compliance $(\mathrm{C})$, weight $(w)$, shear center position $\left(\mathbf{s}_{c}=\left(x_{s}, y_{s}\right)\right)$, and mass center position $\left(\mathbf{m}_{c}=\left(x_{c}, y_{c}\right)\right)$, respectively. The compliance and shear center position values are obtained with the penalized densities, i.e., $p=p_{\max }$.

\begin{tabular}{|c|c|c|c|c|c|c|c|c|c|c|c|c|}
\hline \multirow{2}{*}{ Ref. } & \multirow{2}{*}{$\begin{array}{c}\text { Number of } \\
\text { d.o.f.'s }\end{array}$} & \multirow{2}{*}{$\begin{array}{c}\text { Number of } \\
\text { D.V.'s }\end{array}$} & \multicolumn{2}{|c|}{ Penalization } & \multicolumn{3}{|c|}{ Constraints } & \multirow{2}{*}{$\begin{array}{l}\text { Obj. Func. } \\
\text { eval.'s }\end{array}$} & \multirow{2}{*}{$\mathrm{C}$} & \multirow{2}{*}{$w$} & \multirow[b]{2}{*}{$\mathbf{s}_{c}$} & \multirow{2}{*}{$\mathbf{m}_{c}$} \\
\hline & & & $\Delta p$ & $p_{\max }$ & $\bar{w}$ & $\overline{\mathbf{s}}_{c}$ & $\overline{\mathbf{m}}_{c}$ & & & & & \\
\hline $\mathrm{S} 1$ & $6624 / 390$ & 19044 & 0.1 & 3 & $1 / 2$ & - & 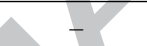 & 1204 & 15.24 & $1 / 2$ & - & - \\
\hline $\mathrm{S} 2$ & $6624 / 390$ & 19044 & 0.1 & 3 & $1 / 2$ & - & - & 1536 & 19.99 & $1 / 2$ & - & - \\
\hline S3 & $6624 / 390$ & 19044 & 0.1 & 3 & $1 / 2$ & - & 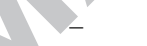 & 1134 & 43.81 & $1 / 2$ & - & - \\
\hline $\mathrm{S} 4$ & $6624 / 390$ & 19044 & 0.1 & 3 & $1 / 2$ & $\begin{array}{l}\bar{x}_{s}=0, \\
\bar{y}_{s}=0\end{array}$ & $\begin{aligned} \bar{x}_{c} & =0, \\
\bar{y}_{c} & =0\end{aligned}$ & 1193 & 44.34 & $1 / 2$ & $\begin{array}{l}x_{s}=0, \\
y_{s}=0\end{array}$ & $\begin{array}{l}x_{c}=0, \\
y_{c}=0\end{array}$ \\
\hline $\mathrm{L} 1$ & $6480 / 390$ & 18216 & 0.1 & 3 & $1 / 2$ & & 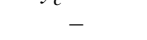 & 1252 & 8.28 & $1 / 2$ & - & - \\
\hline $\mathrm{L} 2$ & $6480 / 390$ & 18216 & 0.1 & 3 & $1 / 2$ & - & - & 1316 & 8.06 & $1 / 2$ & - & - \\
\hline L3 & $6480 / 390$ & 18216 & 0.1 & 3 & & - & - & 1067 & 7.06 & $1 / 2$ & - & - \\
\hline $\mathrm{L} 4$ & $6480 / 390$ & 18216 & 0.1 & 3 & & & $\begin{array}{c}0 \leq \bar{x}_{c} \leq 1 \\
0 \leq \bar{y}_{c} \leq 1\end{array}$ & 1291 & 8.33 & $1 / 2$ & $\begin{array}{c}x_{s}=0.009 \\
y_{s}=0\end{array}$ & $\begin{array}{c}x_{c}=0.60 \\
y_{c}=1\end{array}$ \\
\hline W1 & $6768 / 390$ & 18900 & 0.075 & 8.5 & $1 / 3$ & - & - & 3158 & 13.98 & $1 / 3$ & - & - \\
\hline W2 & $6768 / 390$ & 18900 & 0.075 & & $1 / 3$ & $\bar{x}_{s} \geq-0.2$ & $\bar{x}_{c} \leq 0.1$ & 2784 & 14.62 & $1 / 3$ & $\begin{array}{c}x_{s}=-0.119 \\
y_{s}=0.016\end{array}$ & $\begin{array}{c}x_{c}=0.1, \\
y_{s}=0.013\end{array}$ \\
\hline
\end{tabular}




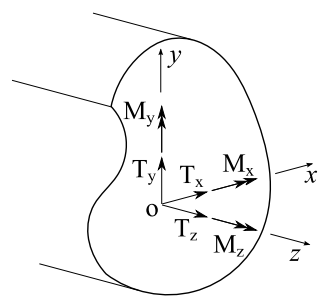

(a) Forces and moments

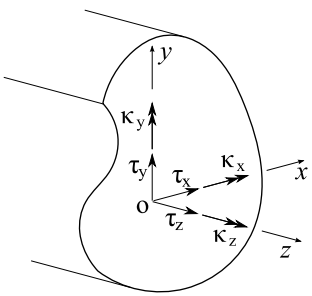

(b) Strains and curvatures
Figure 1: Section coordinate system, forces and moments (a) and corresponding strains and curvatures (b).

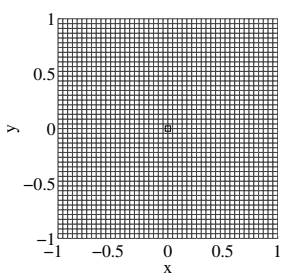

(a) Square

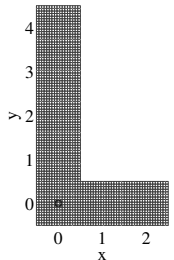

(b) L-shape

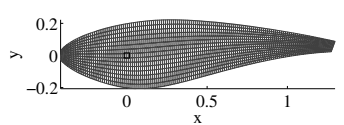

(c) Profile
Figure 2: Cross section dimensions and finite element discretization. (a) Square cross section with 2116 elements, (b) L-shape cross section with 2023 elements, and (c) wing profile with 2100 elements. The square marker indicates the position of the beam node or point of application of the loads.

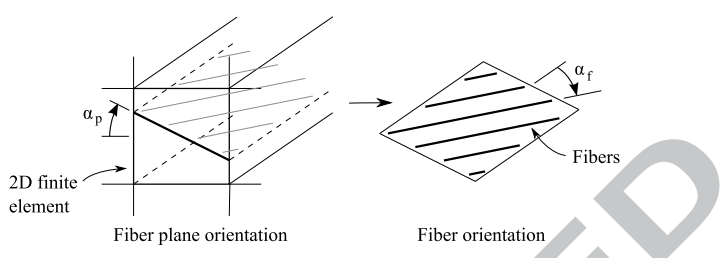

Figure 3: Three-dimensional rotation of fiber plane and fiber orientation in the cross section mesh. The fiber plane orientation is defined by the angle $\alpha_{p}$ while the orientation of the fibers in the fiber plane are defined by the angle $\alpha_{f}$. (a)

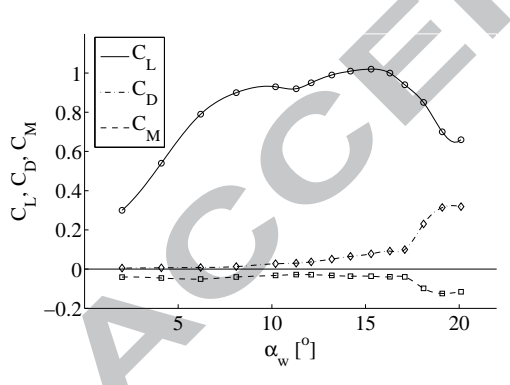

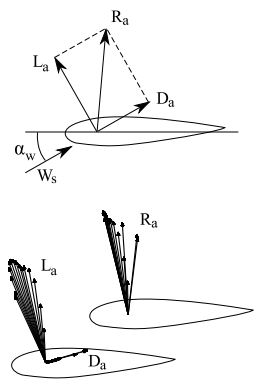

(b)
Figure 4: (a) Aerodynamic lift, drag, and moment coefficients $-C_{L}, C_{D}$, and $C_{M}$, respectively - as function of the angle of attack $\alpha_{w}$. Experimental values for the S809 wind turbine airfoil (Ramsay et al. [35]). (b) Description, magnitude, and orientation of aerodynamic lift and drag forces, $L_{a}$ and $D_{a}$, and corresponding resultant force $R_{a}$, generated by a profile exposed to a wind speed $W_{s}$ at an angle of attack $\alpha_{w}$ for each of the 15 load cases applied in the optimization procedure.

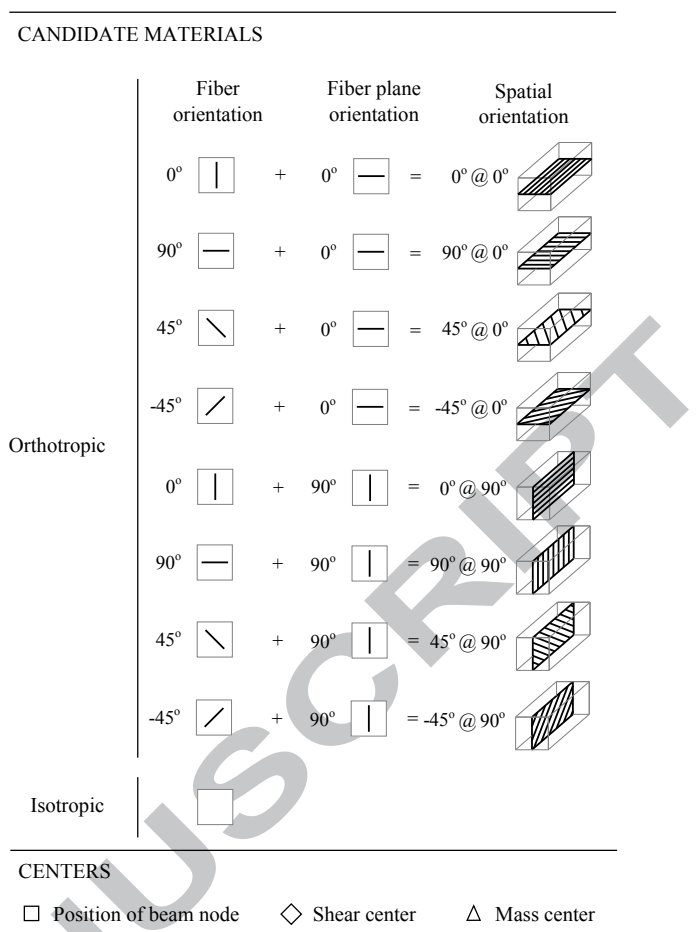

Figure 5: Legend for the figures depicting the optimized cross section designs. (Top) Visualization of the spatial orientation of the fibers at each element based on the resulting fiber and fiber plane orientations, for each of the candidate orthotropic materials. (Bottom) The symbols used to indicate the beam node, shear center and mass center positions can be seen on the right.

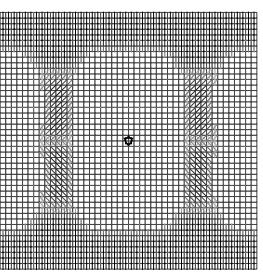

(a) S1 - Fiber

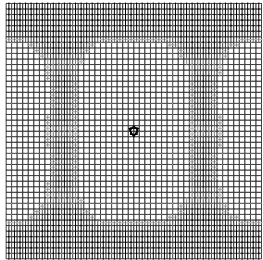

(d) S1 - Fiber plane

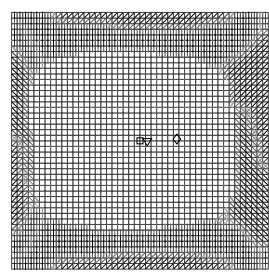

(b) S2 - Fiber

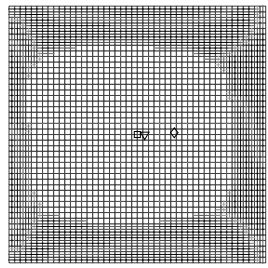

(e) S2 - Fiber plane

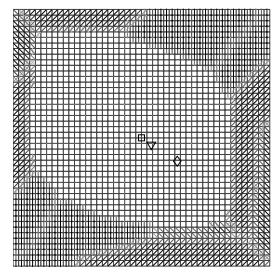

(c) S3 - Fiber

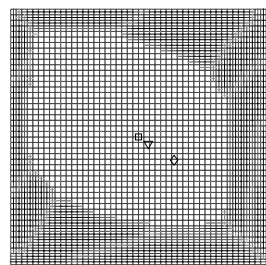

(f) S3 - Fiber plane
Figure 6: Optimal material distribution and laminate properties for square cross section. Solution to the minimum compliance problem with a weight constraint (P2). Fiber (a,b, and c) and fiber plane (d,e, and f) orientations according to figure legend described in Figure 5. (a) and (d) beam subject to vertical transverse force $\widehat{f_{y}}$ (case S1, cf. Table 2) with $f_{r}=0.15$, respectively. (b) and (e) beam subject to combined vertical transverse force $\widehat{f}_{y}$ and torsional moment $\widehat{m}_{z}$ (case S2, cf. Table 2) with $f_{r}=0.1$, respectively. (c) and (f) beam subject to combined horizontal transverse force $\widehat{f}_{x}$, vertical transverse force $\widehat{f_{y}}$, and torsional moment $\widehat{m}_{z}$ (case S3, cf. Table 2) with $f_{r}=0.1$, respectively. 


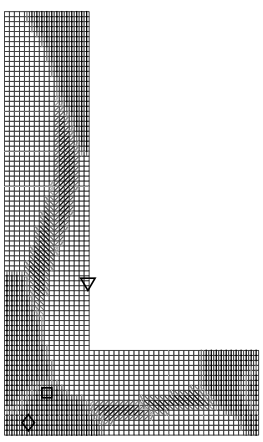

(a) L1 - Fiber

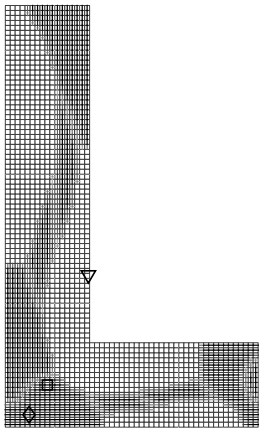

(d) L1 - Fiber plane

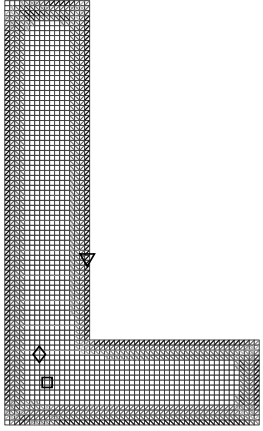

(b) L2 - Fiber

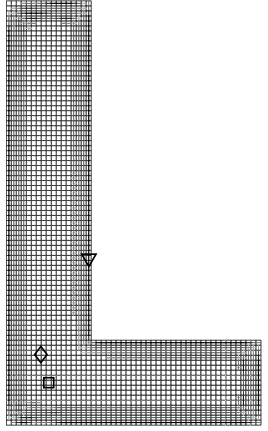

(e) L2 - Fiber plane

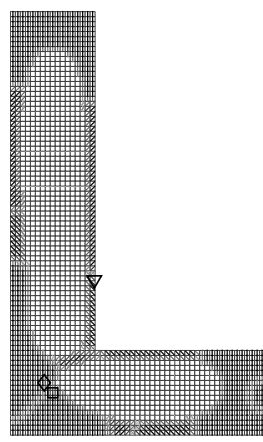

(c) L3 - Fiber

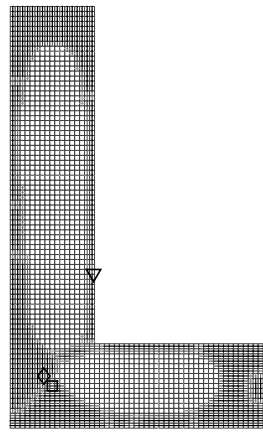

(f) L3 - Fiber plane
Figure 7: Optimal material distribution and laminate properties for L-shape cross section. Solution to the minimum compliance problem with a weight constraint (P2). Fiber (a,b, and c) and fiber plane (d,e, and f) orientations according to figure legend described in Figure 5. (a) and (d) beam subject to combined horizontal transverse force $\widehat{f_{x}}$ and vertical transverse force $\widehat{f_{y}}$ (case L1, cf. Table 2) with $f_{r}=0.125$, respectively. (b) and (e) beam subject to a torsional moment $\widehat{m}_{z}$ (case L2, cf. Table 2) with $f_{r}=0.125$, respectively. (c) and (f) beam subject to horizontal transverse force $\widehat{f}_{x}$ and vertical transverse force $\widehat{f_{y}}$, separately (case L3, cf. Table 2 ), with $f_{r}=0.125$, respectively,

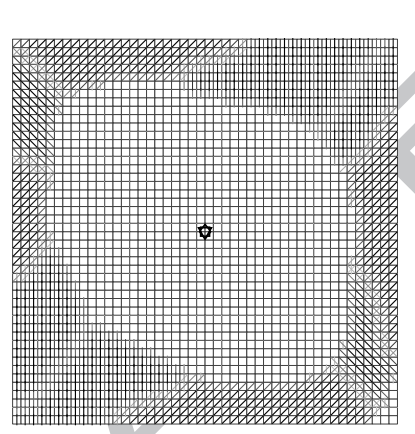

(a) S4 - Fiber

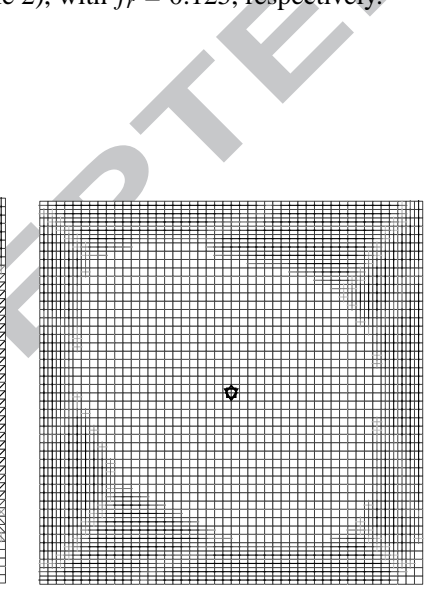

(b) S4 - Fiber plane
Figure 8: Optimal material distribution and laminate properties for square cross section subject to combined horizontal transverse force $\widehat{f}_{x}$, vertical transverse force $\widehat{f_{y}}$, and torsional moment $\widehat{m}_{z}$ (case S4, cf. Table 2) with $f_{r}=0.1$. Solution to the minimum compliance problem with constraints on weight and, shear and mass center positions (P1). Fiber (a) and fiber plane (b) orientations according to figure legend described in Figure 5.

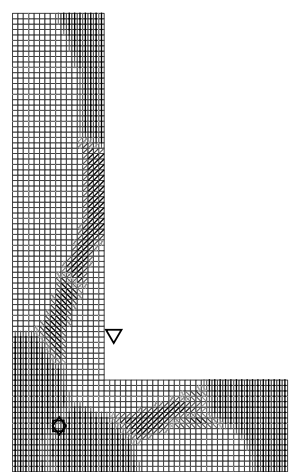

(a) L4 - Fiber

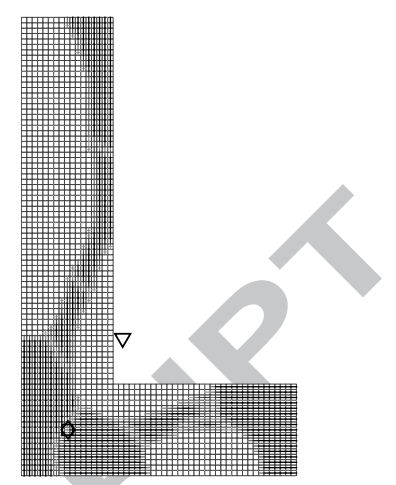

(b) L4 - Fiber plane
Figure 9: Optimal material distribution and laminate properties for L-shape cross section subject to combined horizontal transverse force $\widehat{f_{x}}$ and vertical transverse force $\widehat{f}_{y}$ (case L4, cf. Table 2) with $f_{r}=0.125$. Solution to the minimum compliance problem with constraints on weight and, shear and mass center positions (P1). Fiber (a) and fiber plane (b) orientations according to figure legend described in Figure 5.

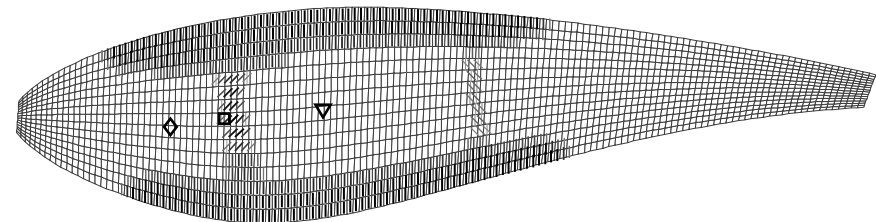

(a) W1 - Fiber

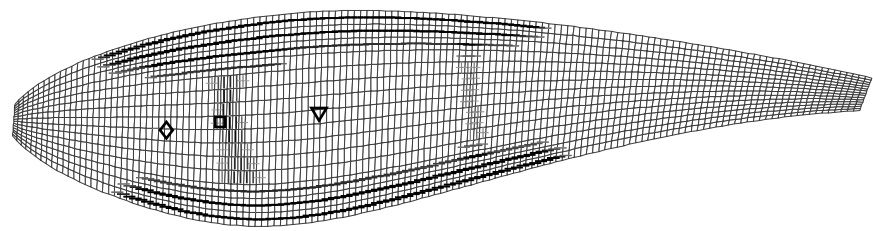

(b) W1 - Fiber plane

Figure 10: Optimal material distribution and laminate properties for the load carrying structure of a wing profile cross section subject to 15 static aerodynamic load cases (case W1, cf. Table 2) with $f_{r}=0.035$. Solution to the minimum compliance problem with a weight constraint (P2). Fiber (a) and fiber plane (b) orientations according to figure legend described in Figure 5. It is assumed that the outer aerodynamic shell is non-structural and exists around the perimeter of the cross section shape outside the design domain. 


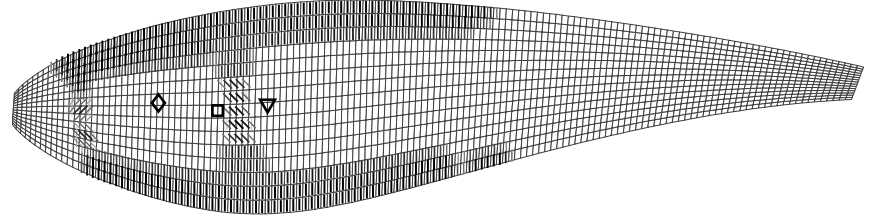

(a) W2 - Fiber

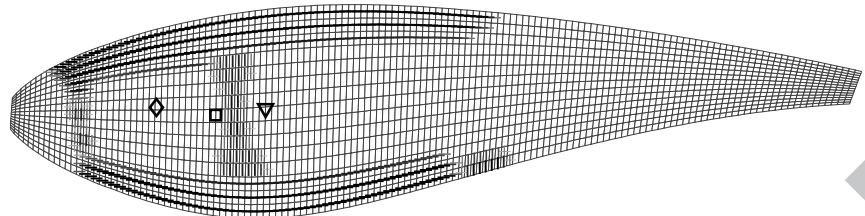

(b) W2 - Fiber plane

Figure 11: Optimal material distribution and laminate properties for the load carrying structure of a wing profile cross section subject to 15 static aerodynamic load cases (case W2, cf. Table 2) with $f_{r}=0.035$. Solution to the minimum compliance problem with constraints on weight and, shear and mass center positions (P1). Fiber (a) and fiber plane (b) orientations according to figure legend described in Figure 5. It is assumed that the outer aerodynamic shell is non-structural and exists around the perimeter of the cross section shape outside the design domain. 